\title{
The influence of stereo information on the mental workload in the manipulator teleoperation process
}

\author{
Weicai Tang ${ }^{1, a}$, Shanguang Chen ${ }^{1,2, b}$, Yi Xiao1 ${ }^{1}$, Guohua Jiang ${ }^{1}$, Zhiqiang Tian ${ }^{1}$ \\ ${ }^{1}$ China Astronaut Research and Training Center, National Key Laboratory of Human Factors \\ Engineering, Beijing 100094, China; \\ ${ }^{2}$ China Manned Space Agency, Beijing 100720, China \\ aemail: jipingacai@163.com, bemail: shanguang_chen@126.com
}

Keywords: teleoperation; stereo information; power spectrum; nonlinear; mental workload

\begin{abstract}
In terms of the specific manipulator teleoperation mission in space station, we divided the teleoperation task into three stages, and then collected electroencephalogram (EEG) and extracted the power spectrum and the nonlinear dynamic characteristics in each stage. Also, to guarantee the task safe and accomplished and improve the evaluation system of mental workload, this paper analyzed the influence of the different stereo information on the mental workload in the three stages of manipulator teleoperation. The results showed that: NASA-TLX can significantly distinguish the change of mental workload of different stereo information levels in teleoperation, power spectrum characteristics of $\theta, \alpha, \beta$ frequency band can effectively evaluate the mental workload level of each stage. In the first two stages, the local and global stereo information had a positive effect on reducing mental workload in teleoperation. On the contrary, the compensation of global stereo information added burden to mental workload in the third stage.
\end{abstract}

\section{Introduction}

Manipulator teleoperation technology plays an important role in space station missions, including astronauts extravehicular operating, on-orbit servicing, goods handling, monitoring, spacecraft docking mission and so on ${ }^{[1]}$. According to the report of NASA, video information based on the terminal camera and the scene camera is the main source of visual information ${ }^{[2]}$. Because of the limit of environment and technology, it is hard to keep the camera information suitable and intact. Thus it brings the cognitive difficulties and the rise of mental workload which has a great influence on the safety and accomplishment of the teleoperation task. There are three stages in teleoperation: in the first stage, astronauts operate mechanical arm until the docking target appears in the vision of the terminal camera. Second, astronauts adjust the position and posture of the load at the end through the terminal camera. Third, astronauts accomplish the task accurately within a specified precision range.

Mental workload refers to the physiological and psychological needs generated by one or more jobs, including the difficulty of task, time stress, effort level, behavior, ability level and many other dimensions ${ }^{[3]}$. The common methods that measure the mental workload contain main-task method, sub-task method, physiological index measurement and subjective questionnaire survey ${ }^{[4][5]}$, and physiological index measurement and subjective questionnaire survey are commonly used now. This research analyzes the influence of different stereo information on the mental workload of the manipulator teleoperation process based on EEG measurement and NASA-TLX survey.

Previous research has found that EEG can reflect the working status. Brouwer and Kohlmorgen extracted the power spectral characteristics of different frequency bands of EEG, then carried out a large number of researches about the mental workload and the difficulty classification of jobs ${ }^{[6][7]}$. Now, $\theta(4 \sim 8 \mathrm{~Hz}), \alpha(8 \sim 13 \mathrm{~Hz})$ and $\beta(14 \sim 30 \mathrm{~Hz})$ three frequency spectrum energy play an important role in mental workload, alertness level, fatigue level, as one of the hotspots of the power spectrum characteristic parameters of EEG. Some studies have found that the three frequency waveform was sensitive to the mental workload. Alpha waveform was active in the occipital lobe and parietal lobe, and it was the main performance when in quiet status. Beta waveform was active in the frontal and 
parietal lobe, and it performed actively when in high intension of thinking activity, high pressure level, high alertness level and excitement status. Theta waveform was active in the occipital lobe and parietal lobe. And it performed actively with the high frequency waveform inactive when in drowsiness, fatigue and rest status ${ }^{[8]}$. In addition to the power spectrum characteristics, many other studies have shown that the nonlinear dynamic characterize of EEG can effectively describe the complexity and the disorder degree of EEG sequence ${ }^{[9][10]}$. C. Papadelis has found that the nonlinear dynamic characterize such as entropy value can effectively reflect the drivers' activity of EEG before and after fatigue deprivation. Others have also found the similar conclusion ${ }^{[11]}$.

In this paper, according to the requirement of the manipulator teleoperation mission on-orbit, we have extracted the power spectrum characteristics and the nonlinear dynamic characteristics of EEG based on the previous researches. We studied the influence of the different stereo information on mental workload in the three stages of manipulator teleoperation, and explored operators' demand of stereo information in each stage of teleoperation. Furthermore, we investigated the difference of the evaluation of mental workload level among NASA-TLX survey, power spectrum characteristics and nonlinear dynamic characteristics indicators. And it would have an important significance for improving information display, reducing mental workload, ensuring the completion of the teleoperation mission and improving the evaluation system of mental workload.

\section{Methods}

The simulation mechanical arm teleoperation experiment platform is based on V-REP PRO simulation robot software platform. Subjects can control the end of mechanical arm to access the target structure with specified posture within the given accuracy and time requirement, through the translation and posture handle with their left and right hands respectively.

The experiment divided the stereo information into 4 levels (A, B, C, D), and studied the change law of the mental workload in the teleoperation process. There is a terminal camera in level A (stereo information missing completely). There is a terminal camera and a global camera in level B (local stereo information missing). There is a terminal camera and a local camera in level C (global stereo information missing). There is a terminal camera, a global and a local camera in level D (integrated stereo information). Figure 1 has shown the scene feedback information of each kind of camera.

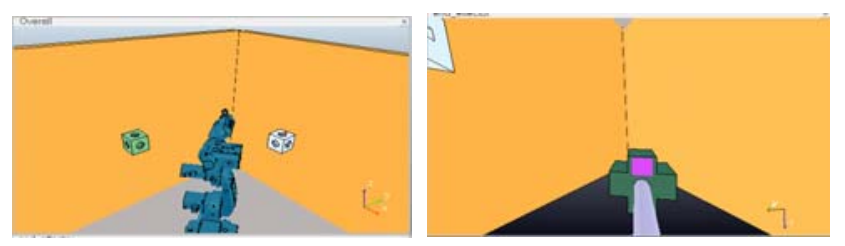

(a) View of global camera (b) View of terminal camera

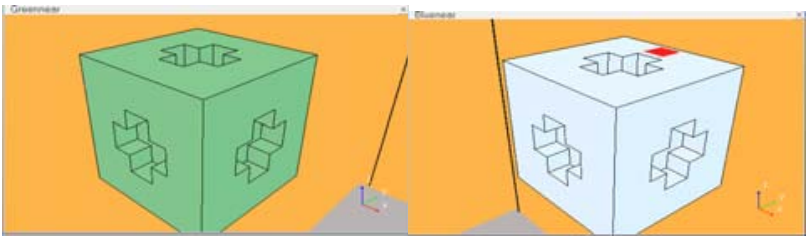

(c) View of local camera

Fig.1. Camera layout

Before the experiment, 24 subjects were accepted the same standard of mechanical arm teleoperation skill training in order to ensure that they had mastered the process of the experiment, the scene of the assignment, handle control and operation strategy. Participants' ages were between 18 and 25, and their vision and body were normal. Formal experiment was carried out in 1 to 3 days after the training. During the formal experiment, EEG signal was acquired by 64 channels BP EEG equipment, and after experiments in each level of stereo information, participants were surveyed by NASA-TLX subjective questionnaire.

\section{Results}

In order to explore the influence of the lack of stereo information on the mental workload in each stage, we extracted the power spectrum characteristics and nonlinear dynamics characteristics of EEG in each stage and carried out the variance analysis aiming at the four information levels in each stage with dividing the EEG signal into 3 stages equally based on the time of operation. 


\section{Power spectrum characteristics}

We carried out the variance analysis aiming at the total power spectrum energy of the four stereo information levels of every channel respectively in each stage. The result showed that: the power spectrum energy of every channel was higher than the others in level A in the first stage. And the four levels showed no significant difference in the second stage. The power spectrum energy of some channels in level A and level D was significant higher than that in level B and C in the third stage.

We extracted P5 channel that is located in the left rear region of the brain and analyzed the change law of power spectrum energy with the frequency band. P5 channel is located in the parietal lobe of cerebral cortex. It is closely relative to the visual perception, spatial information processing, controlling handle and information integration, and it can reflect the influence of the four stereo information levels on the mental workload in the teleoperation process ${ }^{[12]}$. Figure 2 has showed the distribution of the power spectrum energy of P5 channel with frequency band, and 1, 2, 3, 4 correspond to four kinds of frequency band $(\theta, \alpha, \beta, \gamma)$.

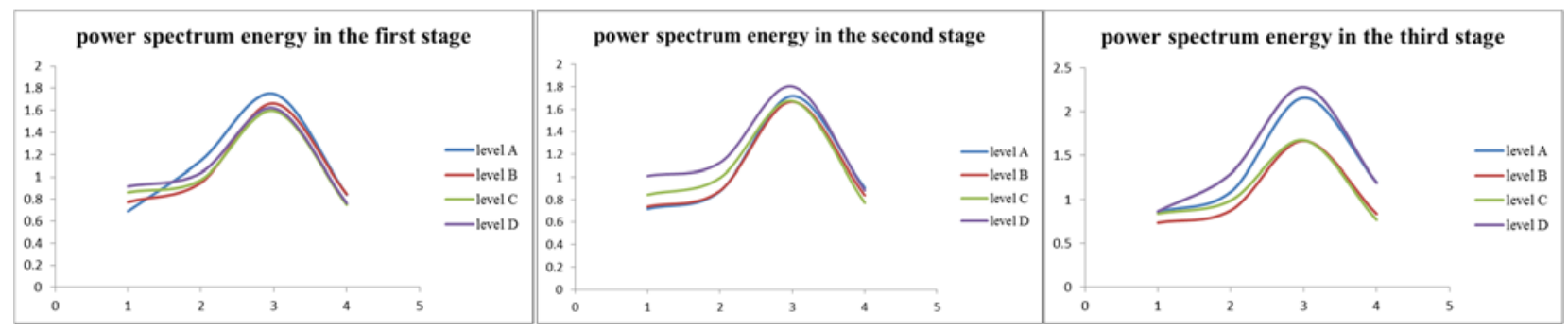

Fig.2. Power spectrum-frequency band curve of P5 channel

As showed in figure 2 , in the $1^{\text {st }}$ stage, power spectrum energy of beta band in level A increased more quickly than in the other three levels. And in the $2^{\text {nd }}$ stage, the energy of theta and alpha band differed obviously except of beta band, and the energy of beta band increased obviously in level A and $\mathrm{B}$ than in level $\mathrm{C}$ and $\mathrm{D}$. In the $3^{\text {rd }}$ stage, energy of beta band differed obviously except of theta band in the four levels, and the energy of beta band increased obviously in level A and D than in level B and C. In addition, compared with the first two stages, the power spectrum energy of beta band in level D increased obviously in the $3^{\text {rd }}$ stage.

To analyze quantitatively the distribution of the energy with the frequency band in the four stereo information conditions, we extracted the power spectrum energy of $\theta, \alpha, \beta$ band respectively and analyzed five characteristic indexes, including $(\alpha+\beta) / \theta, \alpha / \beta,(\alpha+\theta) /(\alpha+\beta),(\alpha+\theta) / \beta, \beta / \theta^{[8]}$. We carried out the single factor variance analysis aiming at the five indexes in the four stereo information conditions respectively in each stage, as shown in table 1.

Table 1 Variance analysis result of the four stereo information levels in each stage

\begin{tabular}{|c|c|c|c|c|c|c|}
\hline \multirow{2}{*}{ mean deviation } & \multicolumn{2}{|c|}{$\mathbf{1}^{\text {st }} \mathbf{s t a g e}$} & \multicolumn{2}{c|}{$\mathbf{2}^{\text {nd }}$ stage } & \multicolumn{2}{c|}{$\mathbf{3}^{\text {rd }}$ stage } \\
\cline { 2 - 6 } & $\mathbf{F}$ & $\mathbf{S i g}$ & $\mathbf{F}$ & Sig & $\mathbf{F}$ & Sig \\
\hline$(\boldsymbol{\alpha}+\boldsymbol{\beta}) / \boldsymbol{\theta}$ & 2.228 & 0.093 & 0.912 & 0.435 & 6.959 & 0.000 \\
\hline $\boldsymbol{\alpha} / \boldsymbol{\beta}$ & 0.144 & 0.934 & 1.759 & 0.155 & 0.703 & 0.551 \\
\hline$(\boldsymbol{\alpha}+\boldsymbol{\theta}) /(\boldsymbol{\alpha}+\boldsymbol{\beta})$ & 1.595 & 0.190 & 2.939 & 0.039 & 2.962 & 0.040 \\
\hline$(\boldsymbol{\alpha}+\boldsymbol{\theta}) / \boldsymbol{\beta}$ & 0.418 & 0.740 & 2.563 & 0.058 & 2.282 & 0.083 \\
\hline $\boldsymbol{\beta} / \boldsymbol{\theta}$ & 1.730 & 0.167 & 1.598 & 0.193 & 6.670 & 0.000 \\
\hline
\end{tabular}

As shown in table 1, the ratio of power spectrum energy between high and low frequency band in the four stereo information conditions had different levels of diversity in each stage. The descriptive statistics is shown in figure 3.

As shown in figure 3, the statistical result was similar with that in figure 2 . In the $1^{\text {st }}$ stage, the ratio of power spectrum energy between high and low frequency band in level A was higher than that in the other levels, especially, $(\alpha+\beta) / \theta$ and $\beta / \theta$ indexes showed the significant diversity between level $A$ and level $D(p=0.022, p=0.023)$. In the $2^{\text {nd }}$ stage, the ratio of power spectrum energy between low and high frequency band in level $\mathrm{C}$ and $\mathrm{D}$ was higher than that in levels $\mathrm{A}$ and $\mathrm{B}$, especially, $\alpha / \beta,(\alpha+\theta) /(\alpha+\beta)$ and $(\alpha+\theta) / \beta(\alpha+\beta) / \theta$ indexes showed the significant diversity between 
level A and level $\mathrm{D}(\mathrm{p}=0.040, \mathrm{p}=0.056, \mathrm{p}=0.015)$. In the $3^{\text {rd }}$ stage, the ratio of power spectrum energy between high and low frequency band in level A and D was higher than that in level $\mathrm{B}$ and $\mathrm{C}$, especially, $(\alpha+\beta) / \theta$ and $\beta / \theta$ indexes showed the significant diversity between level A, D and level C $(\mathrm{p}<0.05)$.

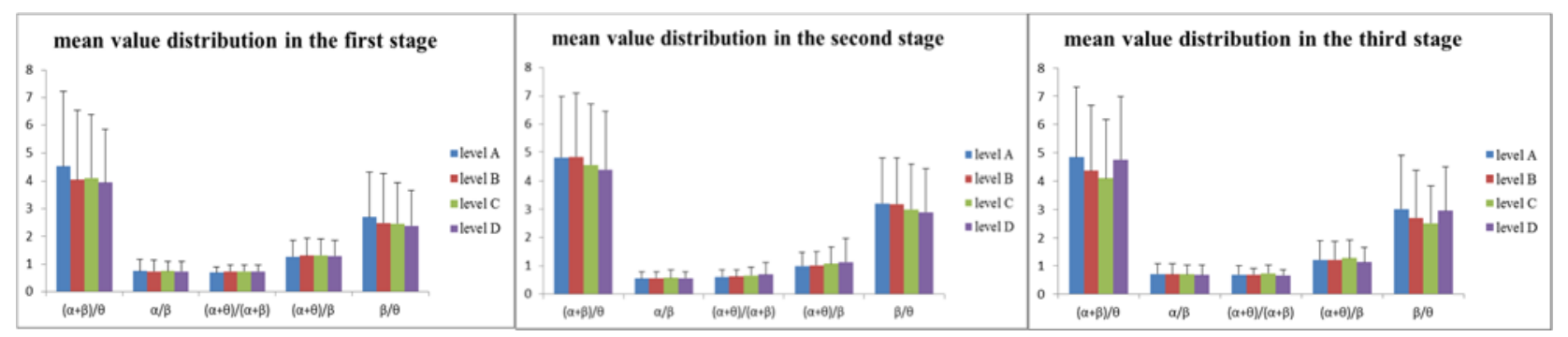

Fig.3. The mean-standard deviation chart of power spectrum characteristics of P5 channel

NASA-TLX subjective questionnaire survey

We carried out the variance analysis of the score of NASA-TLX survey in the four stereo information levels, as shown in figure 4.

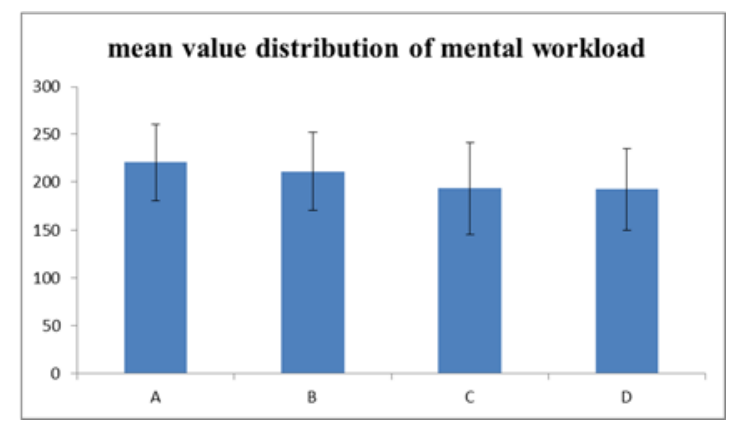

Fig.4. The mean-standard deviation chart of mental workload in the four stereo information levels The paired comparison result of the four levels is shown in table 2 .

Table 2 Paired comparison result of mental load in the four levels

\begin{tabular}{|c|c|c|c|c|c|c|}
\hline mean deviation & A-B & A-C & A-D & B-C & B-D & C-D \\
\hline Mental workload & 9.504 & $26.879^{*}$ & $28.337^{*}$ & 17.375 & 18.833 & 1.458 \\
\hline
\end{tabular}

As shown in figure 4 and table 2, the mental workload score of the four levels showed the obvious diversity. The descending order of the score of the four levels is A, B, C, D, and it showed the significant diversity between level C, D and level A.

\section{Nonlinear dynamic characteristics}

The nonlinear dynamic characteristics of EEG in this paper included Lempel-Ziv repetitive complexity, box-counting fractal dimension and sample entropy. The three kinds of the characteristics can depict the complexity and irregular of the signal effectively. The greater the nonlinear characteristic indexes were, the more complex the time series was, and the more random the signal tended, and the more complicated the dynamic behavior was ${ }^{[13-16]}$.

According to result of the statistical analysis of the nonlinear characteristics, the frontal area showed the better variation trend than the other parts of the cerebral cortex. So we selected AF3, F3, Fp2 channel that is located in the frontal area to study the nonlinear dynamic characteristics. The frontal lobe is closely related with the advanced cognitive function, and it plays an important role in the activity of brain, like learning, language, decision, thinking and so on. Forehead of the brain region ( $\mathrm{Fp} 2$ channel, etc.) is mainly responsible for coordination processing of various regions of the brain, and it is closely related with complex perception, attention, thinking and so on ${ }^{[12]}$. Studying the complexity of the EEG signal by the channels located in the frontal area is helpful for finding the change law. The variance analysis result of the nonlinear characteristics in the four stereo information conditions is shown in table 3.

As shown in table 3, sample entropy of the four stereo information levels in the $1^{\text {st }}$ stage showed the significant diversity. No index showed the significant diversity in the $2^{\text {nd }}$ stage and just the 
reverse in the $3^{\text {rd }}$ stage. The average distribution of the nonlinear characteristics that showed the significant diversity in the four stereo information levels is shown in figure 5.

Table 3 Variance analysis result in the four stereo information conditions in each stage

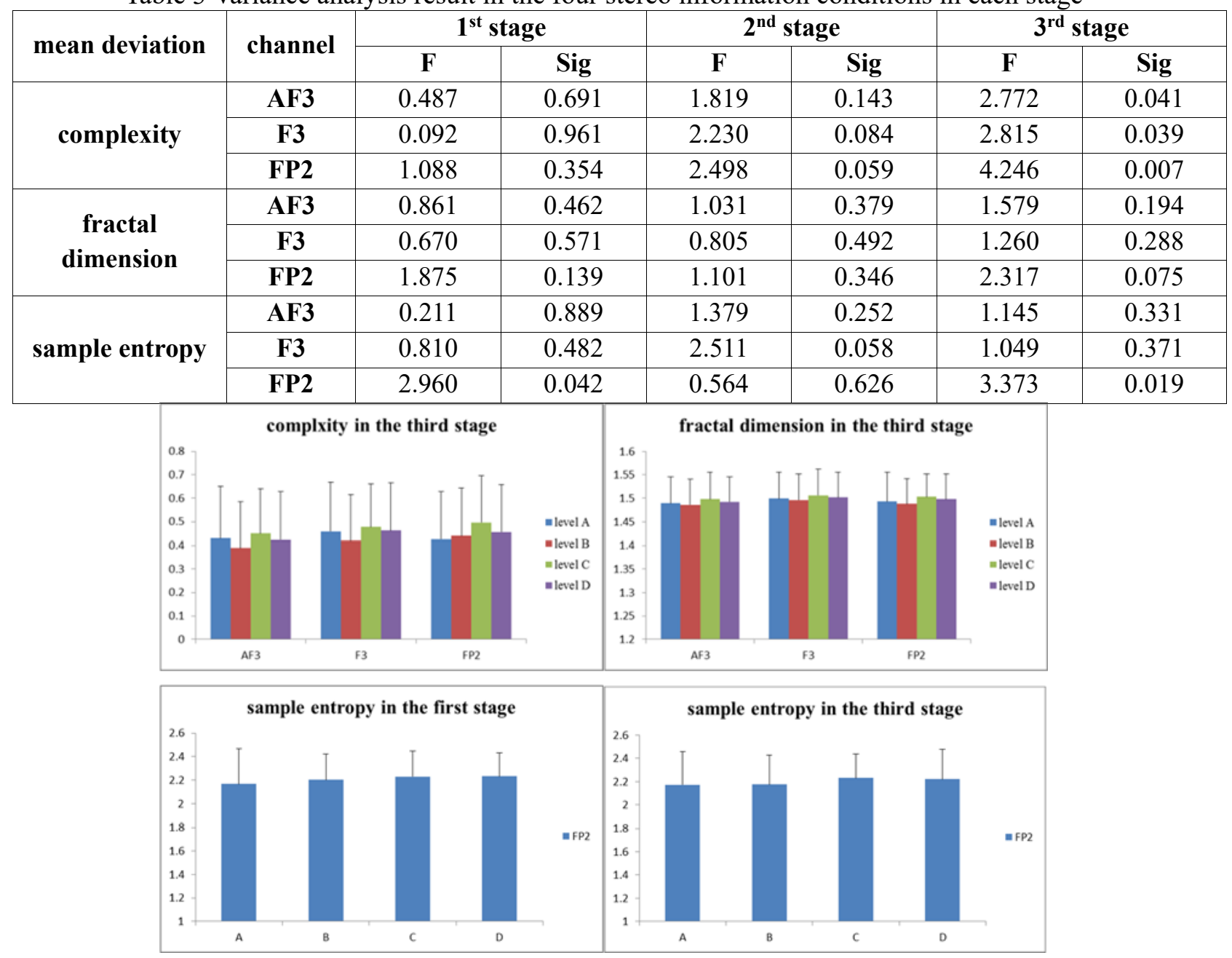

Fig.5. The mean-standard deviation chart of nonlinear characteristic parameter in the four levels The paired comparison result is shown in table 4.

Table 4 Paired comparison result of nonlinear characteristic parameter in the four levels

\begin{tabular}{|c|c|c|c|c|c|c|c|}
\hline mean deviation & channel & A-B & A-C & A-D & B-C & B-D & C-D \\
\hline \multirow{3}{*}{$\begin{array}{c}\text { Complexity in the } \\
3^{\text {rd }} \text { stage }\end{array}$} & AF3 & $0.041(0.074)$ & -0.020 & 0.007 & $-0.061^{*}$ & -0.034 & 0.028 \\
\hline & F3 & $0.040(0.073)$ & -0.019 & -0.003 & $-0.058^{* *}$ & $-0.043^{*}$ & 0.015 \\
\hline & FP2 & -0.014 & $-0.071^{* *}$ & -0.030 & $-0.056^{* *}$ & -0.016 & $0.041^{*}$ \\
\hline \multirow{3}{*}{$\begin{array}{c}\text { fractal dimension } \\
\text { in the } 3^{\text {rd }} \text { stage }\end{array}$} & AF3 & 0.003 & -0.009 & -0.003 & $-0.012^{*}$ & -0.006 & 0.006 \\
\hline & F3 & 0.004 & -0.006 & -0.003 & $-0.010(0.057)$ & -0.006 & 0.004 \\
\hline & FP2 & 0.005 & -0.009 & -0.005 & $-0.014^{*}$ & $-0.010(0.072)$ & 0.004 \\
\hline \multirow{3}{*}{$\begin{array}{l}\text { sample entropy in } \\
\text { the } 1^{\text {st }} \text { stage }\end{array}$} & AF3 & -0.017 & -0.006 & -0.005 & 0.011 & 0.012 & 0.001 \\
\hline & F3 & -0.023 & -0.025 & -0.016 & -0.002 & 0.007 & 0.009 \\
\hline & FP2 & -0.035 & $-0.062^{*}$ & $-0.064^{*}$ & -0.028 & -0.029 & -0.002 \\
\hline \multirow{3}{*}{$\begin{array}{l}\text { sample entropy in } \\
\text { the } 3^{\text {rd }} \text { stage }\end{array}$} & AF3 & -0.009 & -0.025 & $-0.034(0.078)$ & -0.015 & -0.025 & -0.009 \\
\hline & F3 & -0.003 & -0.015 & -0.027 & -0.012 & -0.024 & -0.012 \\
\hline & FP2 & -0.004 & $-0.059^{*}$ & $-0.049(0.055)$ & $-0.056^{*}$ & $-0.046(0.069)$ & 0.010 \\
\hline
\end{tabular}

In the $2^{\text {nd }}$ stage, complexity, fractal dimension, sample entropy showed no significant diversity in the four levels. In the $1^{\text {st }}$ stage, sample entropy in level $\mathrm{C}$ and level D was significant bigger than that in level A. In the $3^{\text {rd }}$ stage, complexity, fractal dimension, sample entropy in level C and D was significant bigger than that in level $\mathrm{A}$ and $\mathrm{B}$. 


\section{Discussion}

\section{Power spectrum characteristics analysis}

According to the previous research, the higher the ratio of the high frequency energy was, the higher operators' mental workload was, and the more the demand of the task was ${ }^{[17-19]}$. In the first two stages, the power spectrum energy of beta frequency band in level A was higher than that in the other three levels, and the ratio of the low frequency energy in level D was higher and the ratio of high frequency energy was lower than that in level A, so the mental workload in level A was higher than that in level D. In the $3^{\text {rd }}$ stage, energy of beta frequency band in level A and D was higher than that in level $\mathrm{B}$ and $\mathrm{C}$, and the energy ratio between high and low frequency band was significant higher than level C, so the mental workload in level A and D was higher than that in level C.

In the $1^{\text {st }}$ stage, energy of each frequency band in level A was higher than that in the other levels, and the ratio of high frequency energy was significant higher than that in level D. This is because at the beginning of the task, the cognizance of the global information and location of the target and also the relationship between load and target is directly related to whether you can rapidly plan the path and the strategy of operation. Target information based on the local camera and the posture information and location information based on the global information play an important role in the cognizance of the task scenarios and the state of mechanical arm, it is helpful to reduce the difficulty of the task and the stress in teleoperation, with the operation efficiency increasing. Therefore, the compensation of local and global stereo information makes a significant contribution to reducing the mental workload at the beginning of teleoperation.

In the $2^{\text {nd }}$ stage, operators had preliminary understanding of the relative position between mechanical arm and target. Also they have formulated the corresponding operation scheme. But without the global information, operators were still hard to understand the actual time posture of mechanical arm and the surrounding scene in the teleoperation process. And with the lack of local information, operators had difficulty cognizing the relative position between the end of the mechanical arm and the environment, the distance specially. And it would easily induce accidents including collision, reaching the joint limit and closing to the workspace. Also it would bring more stress to operators with avoiding accidents and troubleshooting. And as the result, the mental workload in level A with the lack of local and global information increased significantly.

In the $3^{\text {rd }}$ stage, operators adjusted the posture of the load through the local and the terminal camera to fulfil the accuracy requirement of the docking task. Compared with level $\mathrm{C}$, local stereo information is missing in level $\mathrm{A}$, so operators must depend on their experience and try in the process with the demand of their skill and their stress increasing. The mental workload in level A was higher than that in level C. In level D, the compensation of global information would scatter operators' attention and waste the cognitive resource analyzing the redundant information. So the operating stress, physical exertion and time stress in level D increased and the mental workload was higher than that in level C.

\section{Nonlinear characteristics analysis}

As the statistics result of the nonlinear characteristics, in the $1^{\text {st }}$ stage, sample entropy in level C and level D was significant bigger than that in level A. In the $1^{\text {st }}$ stage, operators could not confirm the position of target through the terminal camera, and wasted a lot of time trying to seek it in level A. It had little demand of the analysis ability of spatial relation. After the position of target was confirmed, operators planed the path without considering the relative posture and relative angle and the accidents, so it needed less analysis and inference about the spatial relation than that in level $\mathrm{C}$ and D. According to the previous studies, nonlinear characteristics could reflect the complexity of operators' brain and the intension of thinking, the bigger the nonlinear characteristic value were, the more active the activity of brain was, and the higher the intension of thinking was ${ }^{[5][20-23]}$.

In the $2^{\text {nd }}$ stage, there was no significant diversity in the nonlinear characteristics between the four levels. In this stage, target appeared in the view of terminal camera and operators mainly depended on the information obtained by terminal camera adjusting the load to align with the target. Terminal camera has provided the shape, size, shadow, light which can be the basis for operators to judge the relative posture combined with the perspective principle which belonged to the training 
content. So the stereo information provided by the local and global camera had little effect on operating, there was no significant diversity between the four stereo information levels in the intension of thinking and brain ability, so as the complexity of EEG signal.

In the $3^{\text {rd }}$ stage, complexity, fractal dimension, sample entropy in level $\mathrm{C}$ and $\mathrm{D}$ were significant bigger than that in level A and B. Based on the accuracy requirement of the teleoperation, local stereo information was helpful for cognizing the distance between the load the target in the last stage. In this point, it is important to integrate and make full use of the information provided by local and terminal camera for judging if the load has reached the accuracy requirement, and the higher abilities were needed, including processing ability of image information, attention ability and also the patience of operators. In level A and B, operators could mainly depend on their experience to try constantly, so it needed less analysis and inference about the relative position and distance, so as the intension of thinking than that in level C and D.

\section{Relation among power spectrum, nonlinear characteristics and NASA-TLX survey}

By comparing the statistics result of power spectrum parameters and nonlinear dynamic characteristic parameters, we have found that there was an obvious diversity between the two indexes. According the previous researches, the power spectrum energy of theta, alpha and beta frequency band can comprehensively reflect the change law of operators' mental workload effectively. In NASA-TLX subjective questionnaire, mental workload can divide into six dimensions, including time stress, performance stress, physical exertion, operators' effort, mental demand and frustration level ${ }^{[24]}$. As the analysis result, in level $\mathrm{A}$, the mental workload mainly embodied in difficulty of the task, performance stress, time stress, physical exertion and operators' effort, and it was obviously higher than that in level C and D correspondingly. On the contrary, because of the compensation of local and global stereo information, operators needed to adequately excavate their spatial cognitive resource to integrate the information and plan an efficient strategy, and it required operators keeping their cognitive activity continuous and in the high level, so as the mental activity and intension of thinking increased immensely. Accordingly, the nonlinear dynamic characteristics in level $\mathrm{C}$ and $\mathrm{D}$ were significant higher than that in level A. Nonlinear dynamic characteristics can reflect the intension of thinking and mental, and it can be used as part of the evaluation index of the mental workload.

By comparing the statistics result of power spectrum parameters and NASA-TLX subjective questionnaire score, we have found that the energy ratio between high and low frequency band was mainly identical with NASA-TLX score, the bigger the ratio was, the higher the score of the questionnaire was. While in the $3^{\text {rd }}$ stage, power spectrum energy and the ratio between high and low frequency band showed a higher level in level D, but the score of NASA-TLX survey was not. NASA-TLX survey reflected the mental workload of the whole task, while the power spectrum can divide into three stages and evaluated the mental workload in each stage. And this is the root reason of the difference between the two indexes. In the first two stages, global stereo information has provided the environment information and the relative information between the target and the mechanical arm, and it was helpful for the spatial cognition and operation. But in the $3^{\text {rd }}$ stage, global stereo information has gained the redundant information, so as operators' stress and effort. So in the $3^{\text {rd }}$ stage, the power spectrum parameters can evaluate the mental workload accurately and effectively. Compared with the subjective questionnaire, power spectrum parameters is more flexible.

\section{Conclusion}

NASA-TLX is able to distinguish overall change of the mental workload in teleoperation under different stereo information levels. And the characteristics of EEG power spectrum of $\theta, \alpha, \beta$ frequency band can effectively evaluate the mental workload level in teleoperation. As one of the components of mental workload evaluating indicators, nonlinear dynamics indicators such as box-counting fractal dimension, sample entropy and Lempel-Ziv complexity can reflect the cerebral cortex thinking activities.

In the first two stages, the local and global stereo information had a positive effect on reducing 
mental workload level in operation. On the contrary, the compensation of global stereo information added burden to mental workload in the third stage.

In the $1^{\text {st }}$ and the $3^{\text {rd }}$ stages, the local and global stereo information had increased the intension of mental activity and thinking. While in the $2^{\text {nd }}$ stage, operators mainly depended on the terminal camera and there was no obvious difference among the four stereo information levels.

\section{Acknowledgement}

In this paper, the research was sponsored by the Equipment Advance Research Project (Project No. 51326050204).

\section{References}

[1] Zhang W H, Ye X P, Ji X M, et al. Development summarizing of space robot technology national and outside[J]. Flight Dynamics, 2013, 31(3): 198-202.

[2] Lapointe J F, Dupuis E, Hartman L, et al. An analysis of low-earth orbit space operations[C]//Proceedings of the Joint Association of Canadian Ergonomists/Applied Ergonomics Conference. Alberta, 2002: 1-5.

[3] Sun X S, Guo S Z. Mental Load and Assessment[J]. Science of Social Psychology, 2009(3): $36-40$.

[4] Cui K, Sun L Y, Feng T W, et al. New Developments in Measurement Methodologies of Mental Workload[J]. Industrial Engineering Journal, 2008, 11(5): 1-5.

[5] Ming D, Ke Y F, He F, et al. Psychophysiological measures based studies on mental workload assessment and adaptive automation: Review of the last 40 years and the latest developments[J]. Journal of Electronic Measurement and Instrumentation, 2015, 29(1): 1-13.

[6] Brouwer A M, Hogervorst M A, Van Erp J B F, et al. Estimating workload using EEG spectral power and ERPs in the n-back task[J]. Journal of Neural Engineering, 2012, 9(4): 045008.

[7] Kohlmorgen J, Dornhege G, Braun M, et al. Improving human performance in a real operating environment through real-time mental workload detection[J]. Toward Brain-Computer Interfacing, 2007: 409-422.

[8] Yan Y J. The research of comprehensive evaluation index of driving fatigue based on EEG and cerebral flow[D]. Northeastern University, 2012.

[9] Cao R. Nonlinear and complex network theory in the application of EEG data analysis research[D]. Taiyuan University of Technology, 2014.

[10] Wu X B, Xu J H. Complexity and Brain Function[J]. Biophysics Reports, 1991, 7(1): 103-103.

[11] Liu J, Zhang C, Zheng C. EEG-based estimation of mental fatigue by using KPCA-HMM and complexity parameters[J]. Biomedical Signal Processing and Control, 2010, 5(2): 124-130.

[12] Yao Z B. Clinical neuroanatomy[M]. BEI JING: World Book Incorporated, 2001, 20-24.

[13] Xiao Y, Chen S G, Hang D X, et al. EEG Complexity Method Based on Multiple Coarse-Grained Sequences[J]. Journal of University of Electronic Science and Technology of China, 2013, 42(3): 470.

[14] Zhang D. Research and application of scale division method in Lempel-Ziv complexity[D]. Taiyuan University of Technology, 2013.

[15] Zhang T F, Wu Y Q. Research on Two Fractal Box Dimension[J]. Journal of Ji Lin Jian Zhu University, 2013, 30(4): 84-85. 
[16] Li L, Cao R, Xiang J. Comparative study of approximate entropy and sample entropy based on characterization of EEG[J]. Computer Engineering and Design, 2014, 35(3): 1021-1026.

[17] Zhao L, Dong Y L, Guo X H. Hybrid brain-computer interface system based on $\alpha$ wave and motor imagery[J]. Journal of Electronic Measurement and Instrumentation, 2014, 28(6): 625-629.

[18] Wang F, Wang S N, Wang X H, et al. Driving fatigue detection based on EEG recognition and vehicle handling characteristics[J]. Chinese Journal of Scientific Instrument, 2014, 35(2): 398-404.

[19] Wang F W, Wang H. EEG characteristic analysis of coach bus drivers in fatigue state[J]. Chinese Journal of Scientific Instrument, 2013, 34(5): 1146-1152.

[20] Hang D X, Zhou C D, Liu Y H. Application of Brain State Related EEG Complexity Measure in Mental Workload Evaluation[J]. Space Medicine \& Medical Engineering, 2001, 14(2): 102-106.

[21] Liu J P, Zheng C X. Measurement of EEG Complexity for Studying Sleep[J]. Journal of Biomedical Engineering, 1996, 13(2): 119-122.

[22] Liu J P, Huang Y G. Measuring EEG Complexity for Studying the State of Mental Load[J]. Journal of Biomedical Engineering, 1997, 14(1): 33-37.

[23] Gu F J, Fan S L. Complexity of EEG in Different Function States[J]. Biophysics Reports, 1994, 10(3): 439-445.

[24] Rubio S, Díaz E, Martín J, et al. Evaluation of subjective mental workload: A comparison of SWAT, NASA - TLX, and workload profile methods[J]. Applied Psychology, 2004, 53(1): 61-86. 